\section{Problemas de comportamento internalizantes e externalizantes e uso de substâncias na adolescência}

\author{
Internalizing and externalizing behavior problems \\ and substance use in adolescence
}

\author{
Problemas de comportamiento internalizantes y \\ externalizantes y consumo de sustancias \\ en la adolescencia
}

Wanêssa Lacerda Poton 1

Ana Luiza Gonçalves Soares 2

Helen Gonçalves 3

\section{Resumo}

Este estudo investigou a relação entre problemas de comportamento externalizantes (PCE), internalizantes (PCI) e desses concomitantemente (PCEI) no início da adolescência (11 anos) e o consumo de substâncias (bebidas alcoólicas, tabaco e drogas ilícitas) aos 15 anos, utilizando dados da coorte de nascimentos de Pelotas, Rio Grande do Sul, Brasil, de 1993. Os problemas de comportamento foram avaliados pelo Strengths and Difficulties Questionnaire (SDQ) aplicado às mães quando os filhos tinham 15 anos. O consumo de substâncias foi avaliado por meio de questionário sigiloso autoaplicado ao adolescente. A associação entre problemas de comportamento e uso de substâncias foi analisada por meio de regressão de Poisson com ajuste robusto da variância. Após ajuste para fatores de confusão, os adolescentes com PCE tiveram maior risco para consumo abusivo de álcool $(R R=1,74$; IC95\%: 1,21; 2,50) e para experimentação ( $R R=1,78$; IC95\%: 1,42; 2,23) e uso de tabaco nos últimos 30 dias $(R R=2,38$; IC95\%: 1,64; 3,45). Os adolescentes com PCI tiveram maior risco de experimentar tabaco $(R R=1,54$; IC95\%: 1,22; 1,93) e de utilizá-lo nos últimos 30 dias $(R R=1,92$; IC95\%: $1,31 ; 2,83)$. Os adolescentes com PCEI apresentaram maior risco para experimentação $(R R=2,24$; IC95\%: 1,$57 ; 3,21)$ e consumo de tabaco nos últimos 30 dias ( $R R=3,01$; IC95\%: 1,63; 5,56), e menor risco de experimentação de álcool aos 15 anos ( $R R=0,72$; IC95\%: 0,55; 0,94). Ações de saúde pública que atuem na redução dos problemas de comportamento no início da adolescência poderão diminuir o consumo de tabaco e de bebidas alcoólicas aos 15 anos.

Comportamento; Saúde Mental; Alcoolismo; Fumar; Drogas Ilícitas

\author{
Correspondência \\ W. L. Poton \\ Departamento de Medicina, Universidade Vila Velha. \\ Av. Comissário José Dantas de Melo 21, Vila Velha, \\ ES 29102-920, Brasil. \\ wanessa.poton@uvv.br \\ 1 Departamento de Medicina, Universidade Vila Velha, Vilha \\ Velha, Brasil. \\ 2 University of Bristol, Bristol, U.K. \\ 3 Programa de Pós-graduação em Epidemiologia, Universidade \\ Federal de Pelotas, Pelotas, Brasil.
}




\section{Introdução}

Os transtornos mentais e o uso de substâncias são frequentes na adolescência (10-19 anos) 1,2 e, muitas vezes, ocorrem concomitantemente, contribuindo para o aumento dos anos de vida perdidos por incapacidade (DALYs) 3,4. Estudos mostram que a experimentação e o início do uso frequente de bebidas alcoólicas, tabaco e drogas ilícitas começam na adolescência 5,6,7 e, apesar de os dados mostrarem uma tendência de declínio no uso dessas substâncias entre adolescentes 8 , ele ainda é recorrente e preocupante. Sabe-se que o consumo de substâncias na adolescência predispõe ao seu uso também na vida adulta, repercutindo em desfechos negativos em saúde, tais como ocorrência de doenças crônicas não transmissíveis (gastrointestinais, cardiovasculares e câncer), e provocando ou intensificando consequências sociais, cognitivas e psicológicas 1,2,9.

Estima-se que cerca de 20\% dos adolescentes apresentam algum problema de saúde mental, sendo os mais frequentes os problemas de comportamento internalizantes (PCI) e os externalizantes (PCE) 1 . Os PCI se caracterizam por humor deprimido e ansiedade, preocupação em demasia, medos e inseguranças, sendo, muitas vezes, pouco evidentes, uma vez que não são externados ${ }^{10}$. Em contraste, os PCE manifestam-se por meio de hiperatividade, impulsividade, comportamento desafiador, desobediência, hostilidade e agressividade 10.

A relação entre problemas de comportamento e uso de substâncias na adolescência 11,12,13,14,15,16 e vida adulta 17,18 tem sido alvo de investigação, e, em geral, os estudos mostram que adolescentes com PCE têm risco aumentado para uso de substâncias na adolescência e início da vida adulta 14,15,16,17,18, porém resultados conflitantes são evidenciados para os PCI 12,14,15,16, fato que merece maior atenção. Além disso, PCI e PCE podem coexistir e interagir na relação com o uso de substâncias 19.

A maior parte dos estudos que investigaram a relação entre PCI e PCE e uso de substâncias na adolescência foram realizados em países de alta renda 12,13,14,15,16. No entanto, no Brasil, desigualdades socioeconômicas se mostraram associadas com o consumo de drogas em adolescentes 20 . Indivíduos pertencentes aos grupos socioeconômicos mais elevados têm maior propensão para iniciar o uso de bebidas alcoólicas e drogas 9,20,21, no entanto, maior risco de uso abusivo e dependência de algumas substâncias são evidenciados nas classes socioeconômicas menos favorecidas 9,20,21. Da mesma forma, o contexto e a posição socioeconômica exercem influência sobre a saúde mental, sendo os indivíduos mais pobres os que possuem maior risco para desenvolver alguns transtornos mentais 20,21,22. $\mathrm{O}$ contexto individual, social e cultural pode influenciar na utilização e resposta ao uso de determinadas substâncias em países de baixa e média renda 2 e na ocorrência de PCE e/ou PCI, sendo, portanto, essa relação importante de ser explorada em diferentes contextos socioculturais e econômicos.

Este estudo, portanto, tem como objetivo investigar a relação entre PCE, PCI e PCEI aos 11 anos e o uso de substâncias (bebidas alcoólicas, tabaco e drogas ilícitas) aos 15 anos, utilizando dados de uma coorte de nascimentos de um município de renda média do Brasil.

\section{Métodos}

Foram utilizados dados da coorte de nascimentos de Pelotas, Rio Grande do Sul, Brasil, de 1993. No referido ano, todos os 5.265 nascimentos ocorridos nas maternidades foram identificados, e as mães que residiam na zona urbana e que aceitaram a participação de seu(s) filho(s) no estudo foram entrevistadas, por entrevistadores treinados, logo após o nascimento $(\mathrm{N}=5.249)$, obtendo-se diversas informações socioeconômicas, demográficas e comportamentais, sobre morbidades, história reprodutiva e assistência pré-natal e ao parto, e suas crianças foram medidas, pesadas e examinadas 23 . Na adolescência, os membros da coorte e suas mães responderam a questionários sobre saúde, comportamento e estilo de vida. Para este estudo, dados do nascimento e da adolescência (11 e 15 anos) foram utilizados. As taxas de acompanhamento perinatal, aos 11 e 15 anos foram 99,7\%, ( $\mathrm{n}=5.265$ ), 87,5\% ( $\mathrm{n}=4.452)$ e $85,7 \%(\mathrm{n}=4.349)$, respectivamente 24 . Não foram observadas diferenças significativas na amostra utilizada neste estudo em comparação à coorte original em relação às características sociodemográficas (renda familiar, escolaridade materna, idade materna e presença de companheiro vivendo com a mãe) (Tabela 1). As análises deste estudo foram restritas aos indivíduos que tinham informações completas sobre problemas de comportamento e consumo de bebidas alcoólicas, tabaco e drogas 
Características dos participantes da coorte com informações completas sobre problemas de comportamento externalizantes avaliado pelos pais aos 11 anos e experimentação de álcool aos 15 anos e comparação com a coorte original. Coorte de nascimentos de Pelotas, Rio Grande do Sul, Brasil, 1993-2008

\begin{tabular}{|c|c|c|c|}
\hline & $\begin{array}{l}\text { Coorte original } \\
\qquad \begin{array}{c}(n=5.249) \\
n(\%)\end{array}\end{array}$ & $\begin{array}{c}\text { Amostra } \\
(\mathrm{n}=3.588) \text { * } \\
n(\%)\end{array}$ & Valor de $p * *$ \\
\hline Renda familiar (quintis) & & & 0,126 \\
\hline 1 (menor) & $1.030(20,0)$ & $664(18,9)$ & \\
\hline 2 & $1.195(23,3)$ & $831(23,6)$ & \\
\hline 3 & $889(17,3)$ & $612(17,4)$ & \\
\hline 4 & $1.002(19,5)$ & $722(20,5)$ & \\
\hline 5 (maior) & $1.021(19,9)$ & $690(19,6)$ & \\
\hline Escolaridade materna (anos) & & & 0,178 \\
\hline $0-4$ & $1.467(28,0)$ & $963(26,9)$ & \\
\hline $5-8$ & $2.425(46,3)$ & $1.736(48,4)$ & \\
\hline $9-11$ & $923(17,6)$ & $610(17,0)$ & \\
\hline$\geq 12$ & $427(8,1)$ & $275(7,7)$ & \\
\hline Idade da mãe (anos) & & & 0,899 \\
\hline$<20$ & $916(17,4)$ & $621(17,3)$ & \\
\hline $20-24$ & $1.447(27,6)$ & $980(27,3)$ & \\
\hline $25-29$ & $1.353(25,8)$ & $904(25,2)$ & \\
\hline $30-34$ & $956(18,2)$ & $685(19,1)$ & \\
\hline$\geq 35$ & $576(11,0)$ & $397(11,1)$ & \\
\hline Marido/Parceiro vivendo com a família & & & 0,824 \\
\hline Sim & $4.600(87,6)$ & $3.164(88,2)$ & \\
\hline Não & $649(12,4)$ & $424(11,8)$ & \\
\hline
\end{tabular}

* O valor das variáveis pode diferir do $\mathrm{N}$ total do acompanhamento devido à perda de acompanhamento ou de informação;

** Teste qui-quadrado de heterogeneidade.

ilícitas. Para evitar causalidade reversa, foram excluídos os adolescentes que haviam consumido bebida alcoólica $(\mathrm{n}=408)$ ou tabaco $(\mathrm{n}=201)$ até os 11 anos, utilizando-se, portanto, casos incidentes. Dessa forma, foram incluídos 3.588, 3.626 e 3.504 adolescentes nas análises de consumo de bebidas alcoólicas, tabaco e drogas ilícitas, respectivamente.

\section{Problemas de comportamento externalizantes e internalizantes}

A versão brasileira do Strengths and Difficulties Questionnaire (SDQ) 25 foi aplicada aos adultos responsáveis (93,7\% deles eram mães) e ao adolescente, quando tinham 11 anos de idade. O SDQ é um questionário de rastreio para problemas de saúde mental e possui 25 itens agrupados em cinco escalas: hiperatividade, problemas emocionais, problemas de conduta, problemas com os colegas e comportamento pró-social. Cada escala possui um escore de classificação singular, que é obtido somando-se as pontuações dos itens que a compõem, podendo variar entre 0 e 10 . As escalas investigadas neste estudo foram: (1) hiperatividade e problemas de conduta que, ao serem somados, compreendem os PCE; e (2) problemas de relacionamento com os colegas e problemas emocionais que, quando somados, compõem os PCI. Para este estudo, o resultado do SDQ aplicado ao responsável foi utilizado, e análises suplementares (apresentadas em material suplementar) foram realizadas com o instrumento aplicado aos adolescentes. Os PCE e PCI foram investigados em duas categorias da distribuição dos escores do SDQ: normal (percentil < 90) e anormal (percentil $\geq 90$ ) 26. Tal ponto de corte permite classificar indivíduos normais e anormais em população de baixo risco 26. 
Os adolescentes foram agrupados em quatro categorias de acordo com o comportamento: (1) sem problemas de comportamento; (2) somente PCE; (3) somente PCI; e (4) PCEI (adolescente com ambos PCE e PCI aos 11 anos).

\section{Consumo de substâncias}

Informação sobre o uso de bebidas alcoólicas, tabaco e drogas ilícitas foi obtida por meio de questionário confidencial autoaplicado no acompanhamento dos 15 anos dos participantes.

\section{- Consumo de bebidas alcoólicas}

As seguintes questões avaliaram o consumo de bebidas alcoólicas e foram consideradas neste estudo: (1) “Alguma vez tu já tomaste bebida de álcool?” (sim; não); (2) "Nos últimos 30 dias, quantos dias tu tomaste bebida de álcool?" (1 a 5 dias; 6 a 9 dias; 10 ou mais dias; todos os dias; não ingeri nos últimos 30 dias; nunca ingeri álcool); (3) “Tu já tomaste algum porre ou ficaste bêbado?” (sim; não). Para o consumo atual de bebida alcoólica (últimos 30 dias), as opções de reposta foram recategorizadas em "sim" (consumiu bebida alcoólica, pelo menos, um dia no último mês) e "não" (não ingeriu bebida alcoólica no último mês ou nunca bebeu).

\section{- Consumo de tabaco}

Para avaliar o consumo de tabaco, duas perguntas foram consideradas: (1) "Alguma vez tu experimentaste fumar cigarros, mesmo uma ou duas fumadas?" (sim; não); e (2) "Nos últimos 30 dias, quantos dias tu fumaste?" (1 a 5 dias; 6 a 9 dias; 10 ou mais dias; todos os dias; não fumei; nunca fumei). Essas possibilidades de resposta foram reagrupadas em "sim" (fumou, pelo menos, um dia no último mês) e “não” (não fumou no último mês ou nunca fumou).

\section{- Consumo de drogas ilícitas}

Uma questão foi utilizada para investigar o consumo de drogas ilícitas: (1) "Tu já experimentaste alguma destas coisas (cola de sapateiro, solvente ou tiner, cocaína, maconha)?”. Para experimentação de drogas ilícitas, foi considerado o uso de quaisquer drogas listadas.

\section{Variáveis de confusão}

As potenciais variáveis de confusão, medidas no nascimento, foram: renda familiar mensal (quintis), escolaridade materna em anos de estudo $(0-4 ; 5-8 ; 9-11 ; \geq 12)$, idade materna em anos $(<20 ; 20-24$; $25-29 ; 30-34 ; \geq 35$ ), presença de companheiro ou marido vivendo com a mãe (sim; não), sexo do adolescente. Aos 11 anos, foram consideradas as seguintes variáveis: cor da pele autorreferida pelo adolescente e classificada em branca ou preta/parda/amarela/indígena; tabagismo materno (sim; não); consumo de bebida alcoólica pela mãe (sim; não); transtorno mental comum materno (sim; não). $\mathrm{O}$ transtorno mental comum materno foi avaliado com a aplicação do Self Report Questionnaire (SRQ-20), que contém vinte questões para rastreamento de transtornos mentais não psicóticos. Neste estudo, foi considerado como tendo transtorno mental comum quando a pontuação foi igual ou superior a oito pontos 27 .

\section{Análise estatística}

Inicialmente, o uso de substâncias e os problemas de comportamento foram descritos conforme as variáveis de confusão, sendo as comparações feitas utilizando o teste qui-quadrado de heterogeneidade para as variáveis binárias e categóricas e o teste qui-quadrado de tendência linear para as categóricas ordinais, quando os resultados sugeriam aumento ou redução conforme mudança das categorias 28 .

Para avaliar a associação entre os problemas de comportamento e o uso de bebidas alcoólicas, tabaco e drogas ilícitas, foi utilizada regressão de Poisson com ajuste robusto da variância nas análises 
brutas e ajustadas. Multicolinearidade foi testada utilizando o fator de inflação da variância, sendo considerada presente quando maior que dez 28.

Considerando as diferenças no uso de substâncias conforme sexo e evidência de efeitos diferentes na associação com os problemas de comportamento 16,17,18, foi investigada possível interação com o sexo na relação entre problemas de comportamento e uso de bebidas alcoólicas, tabaco e drogas ilícitas, a qual não se mostrou evidente ( $\mathrm{p}>0,05) 28$.

As análises foram conduzidas no programa Stata, versão 13.0 (StataCorp LP, College Station, Estados Unidos). O estudo foi aprovado pelo Comitê de Ética em Pesquisa com Seres Humanos da Faculdade de Medicina da Universidade Federal de Pelotas (parecer no 4.06.1.095).

\section{Resultados}

Dos participantes da coorte de nascimentos de 1993 de Pelotas incluídos neste estudo, 51,5\% eram do sexo feminino, 66,4\% referiram cor da pele branca, e a maioria era filho de mulheres jovens $(69,8 \%<30$ anos), com até oito anos completos de estudo $(75,3 \%)$, cujo companheiro residia na casa $(88,2 \%)$ (Tabela 2). Quanto à prevalência de problemas de comportamento aos 11 anos, 8,5\% dos adolescentes tinham PCI somente; 7,3\%, apenas PCE; e 2,6\%, PCEI (Tabela 1). Em relação ao uso de substâncias aos 15 anos, tem-se que: 53,1\% haviam experimentado alguma bebida alcoólica; 21,3\% a consumiram nos 30 dias anteriores à entrevista; e 5,9\% o fizeram de modo abusivo; 13,1\% haviam experimentado tabaco; e 4,5\% referiram tê-lo usado nos 30 dias anteriores à entrevista; a experimentação de drogas ilícitas foi relatada por 1,3\% dos adolescentes.

A incidência de utilização de bebidas alcoólicas, tabaco e drogas ilícitas conforme variáveis socioeconômicas, demográficas e maternas são apresentadas na Tabela 3. A experimentação e o consumo recente de bebida alcoólica foram mais frequentes nas meninas, nos adolescentes de maior renda, cujas mães possuíam maior escolaridade, fumavam e consumiam bebidas alcoólicas. O consumo abusivo de álcool foi maior entre aqueles de famílias no maior quintil de renda, cujas mães tinham algum transtorno mental comum. A experimentação de tabaco e seu uso recente foram mais frequentes entre as meninas, nos adolescentes de cor da pele não branca, pertencentes às famílias de menor renda e que tinham mães com menor escolaridade, com transtorno mental comum e fumantes. A experimentação de drogas ilícitas foi mais frequente em adolescentes de cor da pele não branca, cujas mães eram jovens ( $<20$ anos) ou mais velhas ( $\geq 35$ anos), porém não se mostrou associada aos demais fatores socioeconômicos.

A Tabela 4 demonstra a prevalência de PCE, PCI e PCEI em relação às variáveis socioeconômicas, demográficas e maternas. Os PCE foram mais prevalentes entre os meninos, enquanto as meninas apresentaram mais PCI, e a presença de ambos os problemas de comportamento (PCE e PCI) foi mais prevalente em adolescentes de cor da pele não branca. PCE, PCI e PCEI foram mais prevalentes nos adolescentes com menor renda familiar, cujas mães tinham menor escolaridade, apresentavam transtorno mental comum e eram fumantes. Os filhos de mulheres que não possuíam companheiro morando na mesma casa, cujas mães eram mais jovens, apresentaram mais PCE e PCEI, no entanto, essas diferenças não foram evidenciadas para os PCI.

As incidências de utilização de álcool, tabaco e drogas ilícitas, conforme os grupos de problemas de comportamento, quando avaliados pelo responsável, estão apresentadas na Figura 1. A experimentação (58,6; IC95\%: 52,6; 64,5), o consumo nos últimos 30 dias (26,5; IC95\%: 21,1; 31,9) e o consumo abusivo $(9,8$; IC95\%: 6,2; 13,5) de bebidas alcoólicas foram mais frequentes entre os adolescentes que apresentavam PCE. A experimentação de tabaco $(25,5$; IC95\%: 16,7; 34,3) e seu uso recente $(10,4$; IC95\%: 4,2; 16,6) foram mais frequentes nos adolescentes com PCEI, bem como a experimentação de drogas ilícitas. A incidência de utilização de substâncias conforme os problemas de comportamento referidos pelos adolescentes está apresentada em material suplementar (Figura 2). Embora as incidências tenham sido diferentes, o padrão de utilização de substâncias conforme os problemas de comportamento foi, em geral, semelhante, exceto para a experimentação de álcool, que foi maior entre aqueles que não tinham nenhum problema de comportamento (54,0; IC95\%: 52,2; 55,8).

As associações entre PCE, PCI e PCEI e a incidência de uso de bebidas alcoólicas, tabaco e drogas ilícitas foram semelhantes entre meninos e meninas (valor de p para modificação de efeito $>0,05$ ) e, 
Tabela 2

Características dos participantes avaliados ( $N$ = 3.588). Coorte de nascimentos de Pelotas, Rio Grande do Sul, Brasil, 1993.

\begin{tabular}{|c|c|}
\hline Variáveis & $n *(\%)$ \\
\hline \multicolumn{2}{|c|}{ Variáveis coletadas no perinatal } \\
\hline \multicolumn{2}{|c|}{ Sexo do adolescente } \\
\hline Masculino & $1.741(48,5)$ \\
\hline Feminino & $1.847(51,5)$ \\
\hline \multicolumn{2}{|l|}{ Renda familiar (quintis) } \\
\hline 1 (menor) & $664(18,9)$ \\
\hline 2 & $831(23,6)$ \\
\hline 3 & $612(17,4)$ \\
\hline 4 & $722(20,5)$ \\
\hline 5 (maior) & $690(19,6)$ \\
\hline \multicolumn{2}{|l|}{ Idade da mãe (anos) } \\
\hline$<20$ & $621(17,3)$ \\
\hline $20-24$ & $980(27,3)$ \\
\hline $25-29$ & $904(25,2)$ \\
\hline $30-34$ & $685(19,1)$ \\
\hline$\geq 35$ & $397(11,1)$ \\
\hline \multicolumn{2}{|c|}{ Escolaridade materna (anos) } \\
\hline $0-4$ & $963(26,9)$ \\
\hline $5-8$ & $1.736(48,4)$ \\
\hline $9-11$ & $610(17,0)$ \\
\hline$\geq 12$ & $275(7,7)$ \\
\hline \multicolumn{2}{|c|}{ Presença de companheiro na casa (mãe) } \\
\hline Sim & $3.164(88,2)$ \\
\hline Não & $424(11,8)$ \\
\hline \multicolumn{2}{|c|}{ Variáveis maternas e dos participantes coletadas aos 11 anos } \\
\hline \multicolumn{2}{|c|}{ Transtorno mental comum materno } \\
\hline Sim & $1.089(30,5)$ \\
\hline Não & $2.483(69,5)$ \\
\hline \multicolumn{2}{|l|}{ Tabagismo materno } \\
\hline $\operatorname{Sim}$ & $1.934(53,9)$ \\
\hline Não & $1.653(46,1)$ \\
\hline \multicolumn{2}{|c|}{ Consumo de álcool pela mãe } \\
\hline $\operatorname{Sim}$ & $1.543(45,1)$ \\
\hline Não & $1.881(54,9)$ \\
\hline \multicolumn{2}{|c|}{ Cor da pele dos adolescentes } \\
\hline Branca & $2.374(66,4)$ \\
\hline Preta/Parda/Outras & $1.202(33,6)$ \\
\hline \multicolumn{2}{|c|}{ Problemas de comportamento (avaliado pelo responsável) } \\
\hline Sem problemas & $3.925(81,6)$ \\
\hline PCE & $263(7,3)$ \\
\hline $\mathrm{PCl}$ & $305(8,5)$ \\
\hline PCEI & $95(2,6)$ \\
\hline
\end{tabular}

(continua) 
Tabela 2 (continuação)

\begin{tabular}{lc}
\hline Variáveis & $\mathbf{n}$ * $(\%)$ \\
\hline Variáveis dos adolescentes coletadas aos 15 anos & \\
Experimentação de álcool & \\
$\quad$ Sim & $1.906(53,1)$ \\
Não & $1.682(46,9)$ \\
Consumo de álcool nos últimos 30 dias & \\
Sim & $766(21,3)$ \\
Não & $2.823(78,7)$ \\
Consumo abusivo de álcool & $213(5,9)$ \\
Sim & $3.373(94,1)$ \\
Não & \\
Experimentação de tabaco & $474(13,1)$ \\
Sim & $3.152(86,9)$ \\
Não & \\
Consumo de tabaco nos últimos 30 dias & $163(4,5)$ \\
Sim & $3.455(95,5)$ \\
Não & \\
Experimentação de drogas ilícitas & $47(1,3)$ \\
Sim & $3.457(98,7)$ \\
Não &
\end{tabular}

PCE: problemas de comportamento externalizantes, PCEl: problemas de comportamento externalizantes e internalizantes; PCl: problemas de comportamento internalizantes.

* O número de observações pode não ser semelhante em todas as variáveis devido à perda de informação.

portanto, os resultados são apresentados de forma combinada. Após ajuste para fatores de confusão (Tabela 5), a experimentação de álcool foi menor nos adolescentes com PCEI (RR = 0,72; IC95\%: 0,55; 0,94), e o consumo abusivo de álcool foi maior nos adolescentes com PCE (RR = 1,74; IC95\%: 1,21; 2,50). A experimentação de tabaco foi maior entre os que tinham PCE (RR = 1,78; IC95\%: 1,42; 2,23), PCI (RR = 1,54; IC95\%: 1,22; 1,93) e PCEI (RR = 2,24; IC95\%: 1,57; 3,21). O consumo de tabaco nos últimos 30 dias foi maior entre os adolescentes que tinham PCE (RR 2,38; IC95\%: 1,64; 3,45), PCI $(\mathrm{RR}=1,92$; IC95\%: 1,$31 ; 2,83)$ e PCEI (RR = 3,01; IC95\%: 1,$63 ; 5,56)$.

Quando os problemas de comportamento foram avaliados pelo próprio adolescente, as associações entre PCE e consumo abusivo de álcool, experimentação de tabaco e seu uso nos últimos 30 dias se mantiveram, porém em menor magnitude. Para as associações entre PCI e experimentação e uso de tabaco nos últimos 30 dias e entre PCEI e o fumo recente, a magnitude do efeito foi mais forte, e, para a associação entre PCEI e experimentação de tabaco, a associação se manteve, porém mais fraca quando comparada à avaliação dos pais (Tabela 6).

\section{Discussão}

Este estudo mostrou que considerável proporção de adolescentes havia experimentado algumas substâncias, especialmente bebidas alcoólicas, aos 15 anos, sendo que cerca de um quinto deles a consumiu nos últimos 30 dias da entrevista. O uso abusivo de bebidas alcoólicas, experimentação e uso recente de tabaco foram maiores nos adolescentes com PCE, no entanto, aqueles com PCI também apresentaram maior risco para experimentação e uso recente de tabaco. A presença de ambos os problemas de comportamento (PCEI) conferiu risco aumentado para a experimentação e o uso recente de tabaco, porém associação inversa foi observada para a experimentação de bebidas alcoólicas.

Os resultados deste estudo não diferiram dos de pesquisas realizadas em países de alta renda, que mostram que os PCE na adolescência estão associados ao maior consumo de bebidas alcoólicas 12,13,14 
Tabela 3

Incidência de utilização de álcool, tabaco e drogas ilícitas aos 15 anos, conforme variáveis socioeconômicas, demográficas e maternas. Coorte de nascimentos de Pelotas, Rio Grande do Sul, Brasil, 1993.

\begin{tabular}{|c|c|c|c|c|c|c|}
\hline & $\begin{array}{c}\text { Experimentação } \\
\text { de álcool } \\
n(\%)\end{array}$ & $\begin{array}{l}\text { Consumo de } \\
\text { álcool nos } \\
\text { últimos } 30 \\
\text { dias } \\
\text { n (\%) }\end{array}$ & $\begin{array}{l}\text { Consumo } \\
\text { abusivo de } \\
\text { álcool } \\
\text { n (\%) }\end{array}$ & $\begin{array}{c}\text { Experimentação } \\
\text { de tabaco } \\
\mathrm{n}(\%)\end{array}$ & $\begin{array}{c}\text { Fumo nos } \\
\text { últimos } 30 \\
\text { dias } \\
\text { n (\%) }\end{array}$ & $\begin{array}{c}\text { Experimentação } \\
\text { de drogas } \\
\text { ilícitas } \\
\text { n (\%) }\end{array}$ \\
\hline Sexo do adolescente & $p<0,001$ * & $p=0,003$ * & $p=0,481$ * & $p<0,001$ * & $p=0,001$ * & $p=0,328$ * \\
\hline Masculino & $865(48,9)$ & $344(19,3)$ & $114(6,4)$ & $162(9,0)$ & $60(3,3)$ & $21(1,2)$ \\
\hline Feminino & $1.079(57,4)$ & $438(23,4)$ & $109(5,8)$ & $323(17,1)$ & $107(5,7)$ & $29(1,6)$ \\
\hline $\begin{array}{l}\text { Cor da pele do } \\
\text { adolescente }\end{array}$ & $p=0,410$ * & $p=0,919$ * & $p=0,179$ * & $p=0,003$ * & $\mathrm{p}<0,001$ * & $p=0,048$ * \\
\hline Branca & $1.276(53,6)$ & $508(21,3)$ & $150(6,3)$ & $286(11,9)$ & $88(3,7)$ & $25(1,1)$ \\
\hline Preta/Parda/Outras & $628(52,2)$ & $258(21,5)$ & $62(5,2)$ & $188(15,4)$ & $74(6,1)$ & $22(1,9)$ \\
\hline Renda familiar (quintis) & $p<0,001 * *$ & $p<0,001 * \star$ & $p=0,013 * *$ & $p=0,014 * *$ & $p=0,001 * \star$ & $p=0,934 * *$ \\
\hline 1 (menor) & $316(46,5)$ & $123(18,0)$ & $46(6,7)$ & $103(14,9)$ & $40(5,8)$ & $7(1,6)$ \\
\hline 2 & $433(51,4)$ & $170(20,2)$ & $55(6,5)$ & $129(15,2)$ & $46(5,4)$ & $12(1,5)$ \\
\hline 3 & $344(55,4)$ & $129(20,8)$ & $24(3,9)$ & $75(12,0)$ & $31(4,9)$ & $10(1,6)$ \\
\hline 4 & $401(54,9)$ & $160(22,0)$ & $37(5,1)$ & $97(13,2)$ & $26(3,5)$ & $10(1,4)$ \\
\hline 5 (maior) & $421(59,7)$ & $187(26,5)$ & $58(8,2)$ & $70(9,8)$ & $20(2,8)$ & $10(1,4)$ \\
\hline $\begin{array}{l}\text { Escolaridade materna } \\
\text { (anos) }\end{array}$ & $\mathrm{p}<0,001 * *$ & $p<0,001 * *$ & $p=0,091 *$ & $p<0,001 * *$ & $p<0,001 * *$ & $p=0,884 * *$ \\
\hline $0-4$ & $479(48,7)$ & $190(19,3)$ & $65(6,6)$ & $164(16,4)$ & $64(6,4)$ & $16(1,7)$ \\
\hline $5-8$ & $929(52,8)$ & $361(20,6)$ & $95(5,4)$ & $235(13,2)$ & $80(4,5)$ & $22(1,3)$ \\
\hline $9-11$ & $348(56,2)$ & $145(23,3)$ & $37(6,0)$ & $62(10,0)$ & $15(2,4)$ & $22(1,3)$ \\
\hline$\geq 12$ & $184(64,6)$ & $86(30,1)$ & $26(9,1)$ & $22(7,7)$ & $8(2,8)$ & $4(1,4)$ \\
\hline Idade da mãe (anos) & $p=0,902$ * & $p=0,375$ * & $p=0,846$ * & $p=0,094$ * & $p=0,075$ * & $p=0,016$ * \\
\hline$<20$ & $340(53,8)$ & $143(22,6)$ & $43(6,8)$ & $96(15,0)$ & $33(5,2)$ & $15(2,5)$ \\
\hline $20-24$ & $522(52,3)$ & $194(19,4)$ & $58(5,8)$ & $140(13,8)$ & $50(4,9)$ & $12(1,2)$ \\
\hline $25-29$ & $491(53,2)$ & $196(21,2)$ & $54(5,8)$ & $128(13,7)$ & $44(4,7)$ & $11(1,2)$ \\
\hline $30-34$ & $379(54,6)$ & $154(22,2)$ & $40(5,8)$ & $72(10,3)$ & $27(3,9)$ & $3(0,4)$ \\
\hline$\geq 35$ & $211(52,4)$ & $95(23,5)$ & $28(6,9)$ & $49(12,1)$ & $13(3,2)$ & $9(2,3)$ \\
\hline $\begin{array}{l}\text { Presença do } \\
\text { companheiro na casa } \\
\text { (mãe) }\end{array}$ & $p=0,186$ * & $p=0,820$ * & $p=0,608$ * & $p=0,086$ * & $p=0,957$ * & $p=0,351$ * \\
\hline $\operatorname{Sim}$ & $1.700(52,8)$ & $687(21,4)$ & $199(6,2)$ & $416(12,8)$ & $147(4,5)$ & $42(1,3)$ \\
\hline Não & $244(56,2)$ & $95(21,8)$ & $24(5,6)$ & $69(15,7)$ & $20(4,6)$ & $8(1,9)$ \\
\hline $\begin{array}{l}\text { Transtorno mental } \\
\text { comum materno }\end{array}$ & $p=0,392$ * & $p=0,494$ * & $p=0,002$ * & $\mathrm{p}<0,001$ * & $p<0,001$ * & $p=0,063$ * \\
\hline $\operatorname{Sim}$ & $591(54,3)$ & $241(22,1)$ & $85(7,8)$ & $188(17,0)$ & $70(6,4)$ & $20(1,9)$ \\
\hline Não & $1.309(52,7)$ & $523(21,1)$ & $128(5,2)$ & $286(11,4)$ & $93(3,7)$ & $27(1,1)$ \\
\hline Tabagismo materno & $p=0,012$ * & $p=0,001$ * & $p=0,078$ * & $p<0,001$ * & $p<0,001$ * & $p=0,582$ * \\
\hline Sim & $1.065(55,1)$ & $451(23,4)$ & $127(6,6)$ & $298(15,3)$ & $113(5,8)$ & $27(1,4)$ \\
\hline Não & $841(50,9)$ & $315(19,0)$ & $86(5,2)$ & $176(10,5)$ & $50(3,0)$ & $20(1,2)$ \\
\hline $\begin{array}{l}\text { Consumo de álcool } \\
\text { pela mãe }\end{array}$ & $p<0,001$ * & $\mathrm{p}<0,003$ * & $p=0,161$ * & $p=0,290$ * & $p=0,637$ * & $p=0,356$ * \\
\hline Sim & $884(57,3)$ & $368(23,8)$ & $101(6,6)$ & $217(13,9)$ & $75(4,8)$ & $18(1,2)$ \\
\hline Não & $950(50,5)$ & $369(19,6)$ & $102(5,4)$ & $241(12,7)$ & $85(4,5)$ & $29(1,6)$ \\
\hline
\end{tabular}

* Valor de p derivado do teste qui-quadrado de heterogeneidade;

** Valor de p derivado do teste qui-quadrado de tendência linear. 
Tabela 4

Prevalência dos PCE, PCl e PCEI em relação às variáveis socioeconômicas, demográficas e maternas. Coorte de nascimentos de Pelotas, Rio Grande do Sul, Brasil, 1993.

\begin{tabular}{|c|c|c|c|}
\hline & $\begin{array}{c}\text { PCE } \\
\text { n (\%) }\end{array}$ & $\begin{array}{c}\mathrm{PCl} \\
\mathrm{n}(\%)\end{array}$ & $\begin{array}{l}\text { PCEI } \\
\text { n (\%) }\end{array}$ \\
\hline Sexo do adolescente & $\mathrm{p}<0,001$ * & $\mathrm{p}<0,001$ * & $p=0,297$ * \\
\hline Masculino & $197(11,3)$ & $125(7,5)$ & $60(3,7)$ \\
\hline Feminino & $103(6,0)$ & $206(11,4)$ & $51(3,1)$ \\
\hline Cor da pele do adolescente & $p=0,032 *$ & $p<0,001 *$ & $p=0,052 *$ \\
\hline Branca & $183(7,9)$ & $191(8,2)$ & $64(2,9)$ \\
\hline Preta/Parda/Outras & $112(10,1)$ & $138(12,2)$ & $44(4,2)$ \\
\hline Renda familiar (quintis) & $\mathrm{p}<0,001 * \star$ & $\mathrm{p}<0,001 \star \star$ & $\mathrm{p}<0,001 * \star$ \\
\hline 1 (menor) & $67(10,7)$ & $88(13,6)$ & $34(5,7)$ \\
\hline 2 & $81(10,3)$ & $84(10,6)$ & $32(4,3)$ \\
\hline 3 & $58(10,0)$ & $60(10,3)$ & $21(3,9)$ \\
\hline 4 & $56(8,0)$ & $59(8,4)$ & $13(2,0)$ \\
\hline 5 (maior) & $26(3,8)$ & $37(5,3)$ & $10(1,5)$ \\
\hline Escolaridade materna (anos) & $p<0,001 * \star$ & $p<0,001 * *$ & $p<0,001 * \star$ \\
\hline $0-4$ & $94(10,6)$ & $141(15,1)$ & $48(5,7)$ \\
\hline $5-8$ & $169(10,2)$ & $155(9,4)$ & $53(3,4)$ \\
\hline $9-11$ & $31(5,0)$ & $26(4,2)$ & $9(1,5)$ \\
\hline$\geq 12$ & $6(2,2)$ & $9(3,2)$ & $1(0,4)$ \\
\hline Idade da mãe (anos) & $p<0,001 *$ & $p=0,092$ * & $p=0,001 *$ \\
\hline$<20$ & $81(13,9)$ & $69(12,1)$ & $34(6,4)$ \\
\hline $20-24$ & $82(8,6)$ & $86(9,0)$ & $31(3,5)$ \\
\hline $25-29$ & $76(8,7)$ & $89(10,1)$ & $24(2,9)$ \\
\hline $30-34$ & $33(5,0)$ & $52(7,6)$ & $12(1,9)$ \\
\hline$\geq 35$ & $28(7,3)$ & $35(9,0)$ & $10(2,7)$ \\
\hline Presença de companheiro na casa (mãe) & $p=0,011 *$ & $p=0,596$ * & $p=0,001 *$ \\
\hline Sim & $252(8,3)$ & $291(9,4)$ & $87(3,0)$ \\
\hline Não & $48(12,1)$ & $40(10,3)$ & $24(6,4)$ \\
\hline Transtorno mental comum materno & $p<0,001 *$ & $p<0,001 *$ & $p<0,001 *$ \\
\hline Sim & $148(16,0)$ & $188(19,5)$ & $85(9,9)$ \\
\hline Não & $150(6,0)$ & $141(5,6)$ & $26(1,1)$ \\
\hline Tabagismo materno & $p=0,001 *$ & $p=0,001 *$ & $p=0,039 *$ \\
\hline $\operatorname{Sim}$ & $188(10,3)$ & $205(11,1)$ & $69(4,0)$ \\
\hline Não & $112(6,9)$ & $126(7,7)$ & $42(2,7)$ \\
\hline Consumo de álcool pela mãe & $p=0,530 *$ & $p=0,702$ * & $p=0,995$ * \\
\hline Sim & $133(9,0)$ & $144(9,7)$ & $47(3,4)$ \\
\hline Não & $152(8,4)$ & $170(9,3)$ & $58(3,4)$ \\
\hline
\end{tabular}

PCE: problemas de comportamento externalizantes, PCEI: problemas de comportamento externalizantes e internalizantes; $\mathrm{PCl}$ : problemas de comportamento internalizantes.

* Valor de p derivado do teste qui-quadrado de heterogeneidade;

** Valor de p derivado do teste qui-quadrado de tendência linear.

e tabaco 13,14 nessa fase da vida. Independentemente do nível de desenvolvimento do país, o acesso menos ou mais facilitado para o uso de substâncias tem afetado em algum nível os adolescentes 2,21. As explicações indicam que consumi-las faz parte do desejo contínuo dos adolescentes de quebrar regras sociais e se identificar com alguém ou um grupo que apresenta comportamento semelhante 16,18. Outros estudos sugerem que a associação entre os PCE e uso de algumas substâncias podem ser explicados por mecanismos fisiopatológicos, psicopatológicos, ambientais ou genéticos, que podem 
Figura 1

Incidência de utilização de álcool, tabaco e drogas ilícitas aos 15 anos, conforme os problemas de comportamento avaliados pelo responsável. Coorte de nascimentos de Pelotas, Rio Grande do Sul, Brasil, 1993.

1a) Experimentação de álcool

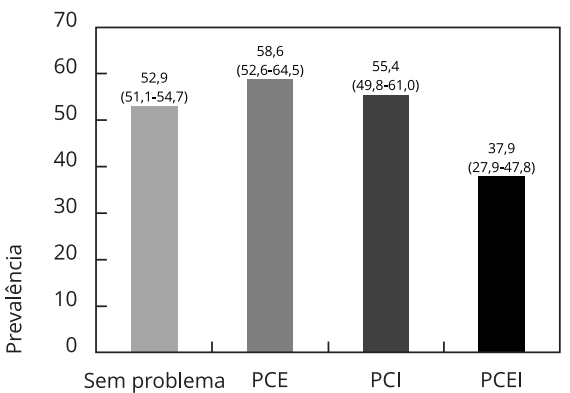

1d) Experimentação de tabaco

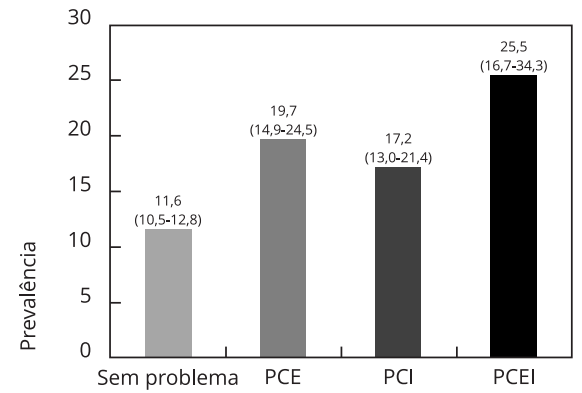

1b) Consumo de álcool nos últimos 30 dias

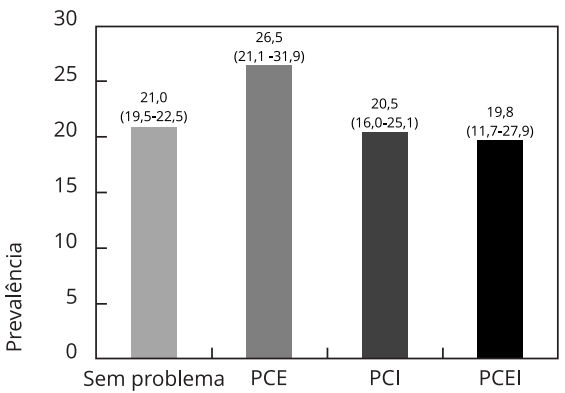

1e) Fumo nos últimos 30 dias

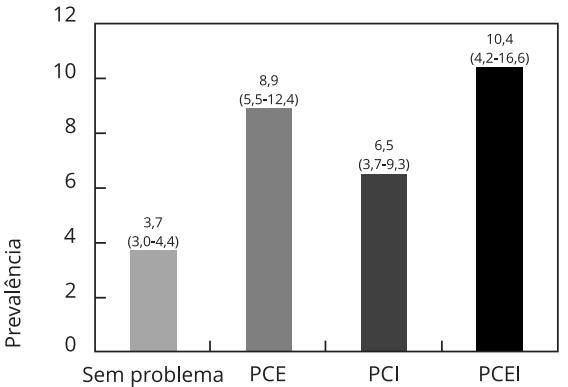

1c) Consumo abusivo de álcool

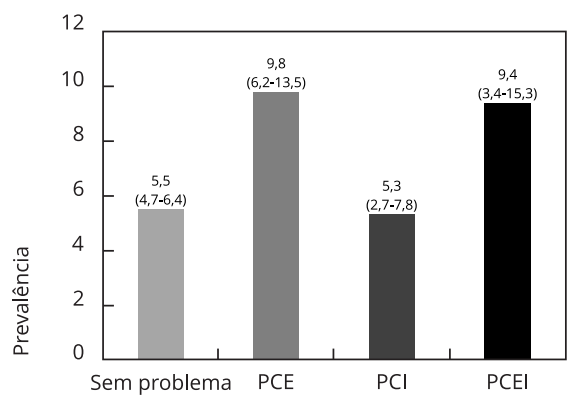

1f) Experimentação de drogas ilícitas

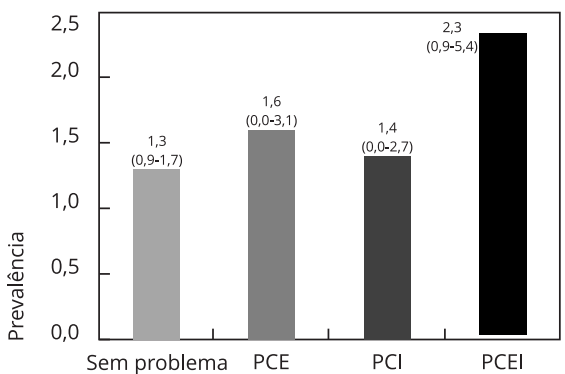

PCE: problemas de comportamento externalizantes, PCEI: problemas de comportamento externalizantes e internalizantes; PCI: problemas de comportamento internalizantes.

estar atuando individualmente ou concomitantemente 18,29,30,31,32,33. Embora a utilização de substâncias investigadas, bem como a prevalência dos PCE e PCI apresentem diferenças em relação ao sexo, neste estudo, não foi observada diferença na associação entre problemas de comportamento e uso de substâncias entre meninos e meninas.

Estudos que avaliam a relação entre PCI e uso de substâncias entre adolescentes apresentam resultados divergentes, não mostrando nenhuma associação ou mostrando tanto associações positivas quanto negativas 12,13,14,15. Uma possível explicação para esse achado é a ocorrência de modificação de efeito dos PCE na relação entre os PCI e uso de substâncias 20,34. Neste estudo, observou-se que os PCI estiveram associados à experimentação e uso recente de tabaco, porém, esse efeito foi potencializado quando os PCE estavam também presentes. Acredita-se que parte dessa associação observada para tabaco, mas não para as demais substâncias, possa ter relação com a alta prevalência no uso de tabaco na região onde este estudo foi realizado 35 , sendo o fumo, portanto, ainda um comportamento socialmente aceito localmente, inclusive entre adolescentes $36 . \mathrm{O}$ consumo de tabaco, especialmente, não requer a presença de um grupo ou a utilização em espaços de lazer, favorecendo que o estado de atenção e a sensação de bem-estar 37 possam ocorrer em mais locais e momentos (caminhadas, na frente do trabalho ou escola) do que o consumo de bebidas alcoólicas e drogas ilícitas. 


\section{Figura 2}

Incidência de utilização de álcool, tabaco e drogas ilícitas aos 15 anos, conforme os problemas de comportamento avaliados pelo adolescente. Coorte de nascimentos de Pelotas, Rio Grande do Sul, Brasil, 1993.

2a) Experimentação de álcool

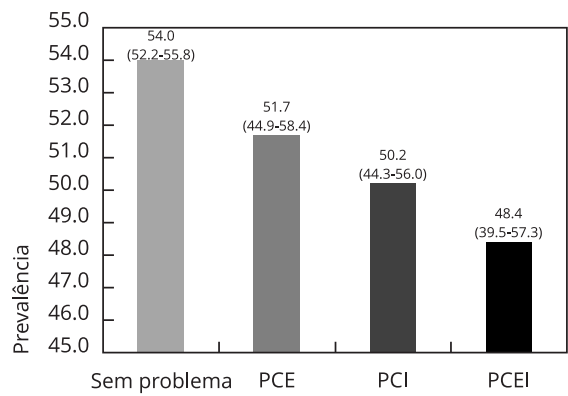

2d) Experimentação de tabaco

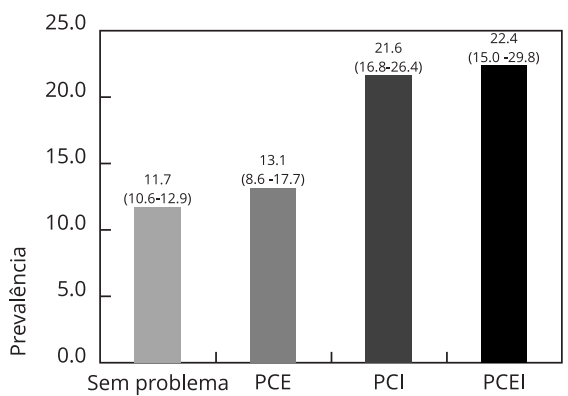

2b) Consumo de álcool nos últimos 30 dias

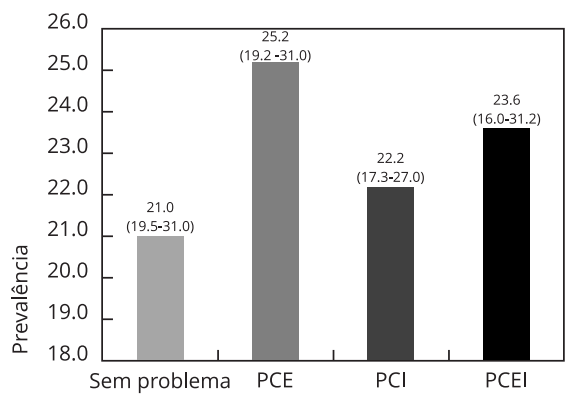

2c) Consumo abusivo de álcool

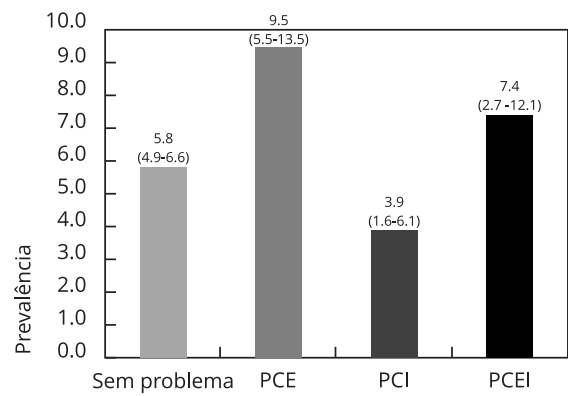

2e) Fumo nos últimos 30 dias

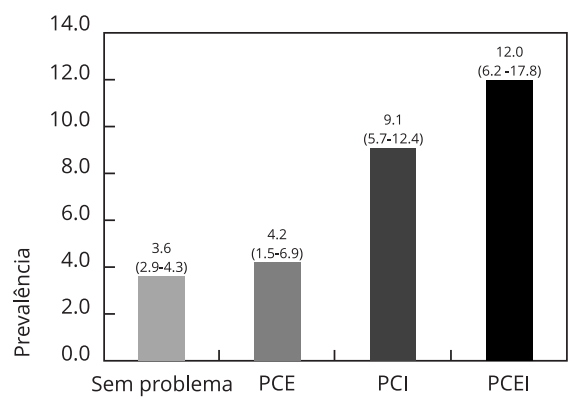

2f) Experimentação de drogas ilícitas

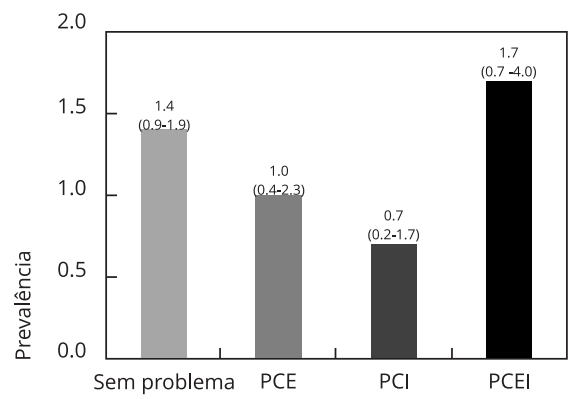

PCE: problemas de comportamento externalizantes, PCEI: problemas de comportamento externalizantes e internalizantes; PCI: problemas de comportamento internalizantes.

Na adolescência, é frequente a presença de PCEI, e a forte influência dos PCE no uso de substâncias pode obscurecer o efeito único dos PCI no uso delas nessa fase da vida ${ }^{34}$. PCE, portanto, podem atuar como moderadores da relação entre os PCI e o uso de substâncias ${ }^{34}$. Neste estudo, encontrou-se aumento no risco para consumo de tabaco entre os adolescentes com PCEI, mas, para o uso bebidas alcoólicas, uma relação inversa foi observada quando o adolescente apresentava PCEI. Colder et al. 19 testaram essa relação entre PCI e PCE e observaram, entre adolescentes norte-americanos - aos 11-12 anos e aos 18-19 anos -, que maiores escores de PCI aumentavam a probabilidade de consumo de bebidas alcoólicas, no entanto, quando os adolescentes tinham também PCE, esse efeito se tornava protetor 19. Outro estudo longitudinal que testou a interação entre os PCI e PCE no início da adolescência (11-12 anos) e o uso de bebidas alcoólicas e drogas (cigarro, maconha e outras drogas ilícitas) aos 13-14 anos encontrou que os adolescentes com altos níveis de PCE e PCI tinham um efeito protetor para o uso dessas substâncias 38 . Estudos que avaliaram a via de mediação desse efeito protetor dos PCI no uso de álcool, quando ocorrendo concomitantemente com os PCE, sugerem que esse problema reduz o envolvimento social do adolescente com amigos que tenham comportamentos considerados desviantes, ou seja, aqueles que poderiam facilitar o acesso às substâncias e incentivá-lo a usá-las 38 . 
Tabela 5

Análise bruta e ajustada da associação entre problemas de comportamento aos 11 anos, avaliados pelo responsável, e utilização de álcool, tabaco e drogas ilícitas aos 15 anos. Coorte de nascimentos de Pelotas, Rio Grande do Sul, Brasil, 1993.

\begin{tabular}{lcccc}
\hline & $\begin{array}{c}\text { Experimentação } \\
\text { de álcool }\end{array}$ & $\begin{array}{c}\text { Consumo de } \\
\text { álcool nos } \\
\text { últimos } 30 \text { dias }\end{array}$ & $\begin{array}{c}\text { Consumo } \\
\text { abusivo de } \\
\text { álcool }\end{array}$ & $\begin{array}{c}\text { Experimentação } \\
\text { de tabaco }\end{array}$ \\
\hline últimos 30 dias \\
PCE
\end{tabular}

PCE: problemas de comportamento externalizantes, PCEl: problemas de comportamento externalizantes e internalizantes; PCI: problemas de comportamento internalizantes; RR: risco relativo.

Nota: modelo ajustado para renda familiar, escolaridade materna, idade materna, presença do parceiro/marido da mãe vivendo com a família, saúde mental materna, tabagismo materno e consumo de álcool pela mãe, sexo e cor da pele do adolescente.

\section{Tabela 6}

Análise bruta e ajustada da associação entre problemas de comportamento aos 11 anos, avaliado pelo adolescente, e utilização de álcool, tabaco e drogas ilícitas aos 15 anos. Coorte de nascimentos de Pelotas, Rio Grande do Sul, Brasil, 1993.

\begin{tabular}{|c|c|c|c|c|c|c|}
\hline & $\begin{array}{c}\text { Experimentação } \\
\text { de álcool }\end{array}$ & $\begin{array}{l}\text { Consumo de } \\
\text { álcool nos } \\
\text { últimos } 30 \text { dias }\end{array}$ & $\begin{array}{l}\text { Consumo } \\
\text { abusivo de } \\
\text { álcool }\end{array}$ & $\begin{array}{c}\text { Experimentação } \\
\text { de tabaco }\end{array}$ & $\begin{array}{l}\text { Fumonos } \\
\text { últimos } 30 \text { dias }\end{array}$ & $\begin{array}{c}\text { Experimentação } \\
\text { de drogas } \\
\text { ilícitas }\end{array}$ \\
\hline \multicolumn{7}{|l|}{ PCE } \\
\hline RR bruto & $0,96(0,84 ; 1,09)$ & $1,19(0,94 ; 1,52)$ & $1,64(1,06 ; 2,56)$ & $1,12(0,78 ; 1,60)$ & $1,17(0,60 ; 2,28)$ & $0,69(0,17 ; 2,84)$ \\
\hline RR ajustado & $0,97(0,87 ; 1,09)$ & $1,13(0,91 ; 1,40)$ & $1,73(1,18 ; 2,53)$ & $1,37(1,05 ; 1,79)$ & $1,87(1,21 ; 2,86)$ & $0,99(0,36 ; 2,76)$ \\
\hline \multicolumn{7}{|l|}{$\mathrm{PCl}$} \\
\hline RR bruto & $0,93(0,82 ; 1,05)$ & $1,05(0,84 ; 1,33)$ & $0,67(0,37 ; 1,22)$ & $1,84(1,44 ; 2,34)$ & $2,51(1,66 ; 3,80)$ & $0,52(0,13 ; 2,13)$ \\
\hline RR ajustado & $0,90(0,81 ; 1,01)$ & $0,99(0,81 ; 1,22)$ & $0,85(0,54 ; 1,35)$ & $1,84(1,48 ; 2,27)$ & $2,70(1,89 ; 3,85)$ & $0,77(0,28 ; 2,14)$ \\
\hline \multicolumn{7}{|l|}{ PCEI } \\
\hline RR bruto & $0,90(0,74 ; 1,08)$ & $1,12(0,81 ; 1,55)$ & $1,27(0,67 ; 2,43)$ & $1,91(1,35 ; 2,68)$ & $3,33(1,20 ; 5,55)$ & $1,19(0,29 ; 4,87)$ \\
\hline RR ajustado & $0,92(0,76 ; 1,12)$ & $1,03(0,71 ; 1,49)$ & $1,52(0,80 ; 2,88)$ & $2,06(1,45 ; 2,92)$ & $3,81(2,29 ; 6,32)$ & $1,34(0,33 ; 5,48)$ \\
\hline
\end{tabular}

PCE: problemas de comportamento externalizantes, PCEI: problemas de comportamento externalizantes e internalizantes; PCI: problemas de comportamento internalizantes; RR: risco relativo.

Nota: modelo ajustado para renda familiar, escolaridade materna, idade materna, presença do parceiro/marido mãe vivendo com a família, saúde mental materna, tabagismo materno e consumo de álcool pela mãe, sexo e cor da pele do adolescente.

Alguns pontos positivos e limitações do presente estudo merecem destaque. Um ponto positivo é o fato de ser um estudo longitudinal, permitindo examinar a relação prospectiva entre os PCE, PCI e PCEI aos 11 anos e o uso de substâncias aos 15 anos. Para evitar causalidade reversa, os adolescentes que haviam experimentado bebidas alcoólicas e tabaco até os 11 anos foram excluídos das análises. As informações sobre o consumo de bebidas alcoólicas, tabaco e drogas ilícitas foram coletadas por meio de um questionário anônimo autoaplicado, o que pode ter reduzido a subestimação da incidência de 
uso dessas substâncias. A aplicação do instrumento de avaliação dos problemas de comportamento ao responsável, no entanto, pode ter subestimado a prevalência dos PCI e superestimado os PCE, e a relação entre os problemas de comportamento e o uso de substâncias pode diferir dependendo do informante (quem responde ao questionário) 12. Foram realizadas, portanto, análises suplementares com as informações do SDQ respondidas pelos adolescentes aos 11 anos, as quais mostraram redução do efeito observado para os PCE e leve aumento na magnitude dos efeitos para os PCI quando comparados aos resultados obtidos na avaliação do responsável.

Este estudo mostrou que, além dos PCE, os PCI também estiveram associados à experimentação e ao uso recente de tabaco, porém não com as demais substâncias avaliadas, e a presença de PCEI apresentou um risco mais elevado para o consumo de tabaco. Intervenções destinadas à identificação precoce de adolescentes com problemas de comportamento, que atuem na promoção da consciência sobre as consequências à saúde do consumo de substâncias, poderão minimizar os riscos nessa fase da vida.

\section{Colaboradores}

W. L. Poton conduziu o trabalho, participando da concepção, análise, interpretação dos dados, redação do manuscrito e sua aprovação final. A. L. G. Soares e H. Gonçalves participaram da concepção, interpretação dos dados, redação e revisão crítica do manuscrito para sua aprovação final.

\section{Agradecimentos}

Este artigo foi realizado com dados do estudo Coorte de Nascimentos de Pelotas, Rio Grande do Sul, Brasil, em 1993, conduzido pelo Programa de Pós-graduação em Epidemiologia da Universidade Federal de Pelotas, com o apoio da Associação Brasileira de Saúde Coletiva (ABRASCO). De 2004 a 2013, a coorte de nascimentos de 1993 foi financiada pelo Wellcome Trust. Fases anteriores do estudo foram financiadas pela União Europeia, Programa de Apoio a Núcleos de Excelência (PRONEX), Conselho Nacional de Desenvolvimento Científico e Tecnológico (CNPq) e Ministério da Saúde.

\section{Referências}

1. Currie C, Zanotti C, Morgan A, Currie D, Looze M, Roberts C, et al. Social determinants of health and well-being among young people. Health Behaviour in School-aged Children (HBSC) study: international report from the 2009/2010 survey. Copenhagen: World Health Organization; 2012.

2. Hall WD, Patton G, Stockings E, Weier M, Lynskey M, Morley KI, et al. Why young people's substance use matters for global health. Lancet Psychiatry 2016; 3:265-79.

3. Kieling C, Baker-Henningham $\mathrm{H}$, Belfer M, Conti G, Ertem I, Omigbodun O, et al. Child and adolescent mental health worldwide: evidence for action. Lancet 2011; 378:1515-25.

4. Gore FM, Bloem PJ, Patton GC, Ferguson J, Joseph V, Coffey C, et al. Global burden of disease in young people aged 10-24 years: a systematic analysis. Lancet 2011; 377:2093-102. 
5. Coutinho ESF, França-Santos D, Magliano ES, Bloch KV, Barufaldi LA, Cunha CF, et al. ERICA: padrões de consumo de bebidas alcoólicas em adolescentes brasileiros. Rev Saúde Pública 2016; 50 Suppl 1:8s.

6. Figueiredo VC, Szklo AS, Costa LC, Kuschnir MCC, Silva TLN, Bloch KV, et al. ERICA: smoking prevalence in Brazilian adolescents. Rev Saúde Pública 2016; 50 Suppl 1:12s.

7. Horta RL, Horta BL, Costa AW, Prado RR, Oliveira-Campos M, Malta DC. Lifetime use of illicit drugs and associated factors among Brazilian schoolchildren, National Adolescent School-based Health Survey (PeNSE 2012). Rev Bras Epidemiol 2014; 17 Suppl 1:31-45.

8. Peiper NC, Ridenour TA, Hochwalt B, CoyneBeasley T. Overview on prevalence and recent trends in adolescent substance use and abuse. Child Adolesc Psychiatr Clin N Am 2016; 25:349-65.

9. World Health Organization. Global status report on alcohol and health, 2014. Geneva: World Health Organization; 2014.

10. American Psychiatric Association. DSM-5: Manual diagnóstico e estatístico de transtornos mentais. 5a Ed. Porto Alegre: Editora Artmed; 2014.

11. Heron J, Maughan B, Dick DM, Kendler KS, Lewis G, Macleod J, et al. Conduct problem trajectories and alcohol use and misuse in mid to late adolescence. Drug Alcohol Depend 2013; 133:100-7.

12. McCarty CA, Wymbs BT, King KM, Mason WA, Vander Stoep A, McCauley E, et al. Developmental consistency in associations between depressive symptoms and alcohol use in early adolescence. J Stud Alcohol Drugs 2012; 73:444-53.

13. Maslowsky J, Schulenberg JE. Interaction matters: quantifying conduct problem $\mathrm{x}$ depressive symptoms interaction and its association with adolescent alcohol, cigarette, and marijuana use in a national sample. Dev Psychopathol 2013; 25(4 Pt 1):1029-43.

14. Colder CR, Scalco M, Trucco EM, Read JP, Lengua LJ, Wieczorek WF, et al. Prospective associations of internalizing and externalizing problems and their co-occurrence with early adolescent substance use. J Abnorm Child Psychol 2013; 41:667-77.

15. Parrish KH, Atherton OE, Quintana A, Conger $\mathrm{RD}$, Robins RW. Reciprocal relations between internalizing symptoms and frequency of alcohol use: findings from a longitudinal study of Mexican-origin youth. Psychol Addict Behav 2016; 30:203-8.
16. Jun HJ, Sacco P, Bright CL, Camlin EA. Relations among internalizing and externalizing symptoms and drinking frequency during adolescence. Subst Use Misuse 2015; 50:1814-25.

17. Thompson KD, Leadbeater BJ, Ames ME. Reciprocal effects of internalizing and oppositional defiance symptoms on heavy drinking and alcohol-related harms in young adulthood. Subst Abuse 2016; 9 Suppl 1:21-31.

18. Foster KT, Hicks BM, Lacono WG, McGue M. Gender differences in the structure of risk for alcohol use disorder in adolescence and young adulthood. Psychol Med 2015; 45:3047-58.

19. Colder CR, Frndak S, Lengua LJ, Read JP, Hawk Jr. LW, Wieczorek WF. Internalizing and externalizing problem behavior: a test of a latent variable interaction predicting a twopart growth model of adolescent substance use. J Abnorm Child Psychol 2018; 46:319-30.

20. Centro Brasileiro de Informações sobre Drogas Psicotrópicas. VI Levantamento nacional sobre o consumo de drogas psicotrópicas entre estudantes do Ensino Fundamental e Médio das redes pública e privada de ensino nas 27 capitais brasileiras 2010. São Paulo: Centro Brasileiro de Informações sobre Drogas Psicotrópicas; 2010.

21. United Nations Office on Drugs and Crime. World drug report 2016. Vienna: United Nations; 2016.

22. Ogundele MO. Behavioural and emotional disorders in childhood: a brief overview for paediatricians. World J Clin Pediatr 2018; 7:926.

23. Victora CG, Hallal PC, Araújo CLP, Menezes AMB, Wells JCK, Barros FC. Cohort profile: the 1993 Pelotas (Brazil) Birth Cohort Study. Int J Epidemiol 2008; 37:704-9.

24. Gonçalves H, Assunção MCF, Wehrmeister FC, Oliveira IO, Barros FC, Victora CG, et al. Cohort Profile update: The 1993 Pelotas (Brazil) Birth Cohort follow-up visits in adolescence. Int J Epidemiol 2014; 43:1082-8.

25. Fleitlich B, Cortázar PG, Goodman R. Questionário de capacidades e dificuldades (SDQ). Infanto Rev Neuropsiquiatr Infanc Adolesc 2000; 8:44-50.

26. Goodman R. The Strengths and Difficulties Questionnaire: a research note. J Child Psychol Psychiatry 1997; 38:581-6. 
27. Mari JJ, Williams P. A comparison of the validity of two psychiatric screening questionnaires (GHQ-12 and SRQ-20) in Brazil, using relative operating characteristic (ROC) analysis. Psychol Med 1985; 15:651-9.

28. Kirkwood BR, Sterne JAC. Essential medical statistics. 2nd Ed. London: British Library; 2013.

29. Arcos-Burgos M, Vélez JI, Solomon BD, Muenke M. A common genetic network underlies substance use disorders and disruptive or externalizing disorders. Hum Genet 2012; 131:917-29.

30. Calafat A, García F, Juan M, Becoña E, Fernández-Hermida JR. Which parenting style is more protective against adolescent substance use? Evidence within the European context. Drug Alcohol Depend 2014; 138:185-92.

31. Tyler KA, Handema R, Schmitz RM, Phiri F, Kuyper KS, Wood C. Multi-Level risk and protective factors for substance use among Zambian street youth. Subst Use Misuse 2016; 51:922-31

32. Trucco EM, Hicks BM, Villafuerte S, Nigg JT, Burmeister M, Zucker RA. Temperament and externalizing behavior as mediators of genetic risk on adolescent substance use. J Abnorm Psychol 2016; 125:565-75.

33. Ashenhurst JR, Harden KP, Corbin WR, Fromme K. Alcohol-related genes show an enrichment of associations with a persistent externalizing factor. J Abnorm Psychol 2016; 125:933-45.
34. Hussong AM, Jones DJ, Stein GL, Baucom DH, Boeding S. An internalizing pathway to alcohol use and disorder. Psychol Addict Behav 2011; 25:390-404.

35. Barros AJ, Cascaes AM, Wehrmeister FC, Martínez-Mesa J, Menezes AM. Tobacco smoking in Brazil: regional inequalities and prevalence according to occupational characteristics. Ciênc Saúde Coletiva 2011; 16:3707-16.

36. Barreto SM, Giatti L, Oliveira-Campos M, Andreazzi MA, Malta DC. Experimentação e uso atual de cigarro e outros produtos do tabaco entre escolares nas capitais brasileiras (PeNSE 2012). Rev Bras Epidemiol 2014; 17 Suppl 1:62-76.

37. World Health Organization. Neuroscience of psychoactive substance use and dependence. Geneva: World Health Organization; 2004.

38. Scalco MD, Colder CR, Hawk LW, Read JP, Wieczorek WF, Lengua LJ. Internalizing and externalizing problem behavior and early adolescent substance use: a test of a latent variable interaction and conditional indirect effects. Psychol Addict Behav 2014; 28:828-40. 


\section{Abstract}

This study investigated the relationship between externalizing behavior problems (EBP), internalizing behavior problems (IBP), and combined externalizing/internalizing behavior problems $(E I B P)$ in early adolescence (11 years) and substance use (alcohol, tobacco, and illegal drugs) at 15 years, using data from the 1993 Pelotas (Brazil) birth cohort. Behavior problems were assessed with the Strengths and Difficulties Questionnaire $(S D Q)$, applied to mothers when their children were 15 years old. Substance use was assessed with a self-applied confidential questionnaire for the adolescent. The association between behavior problems and substance use was analyzed with Poisson regression with robust adjustment of variance. After adjusting for confounding, adolescents with EBP showed higher risk of alcohol abuse $(R R=1.74,95 \% C I: 1.21$; 2.50) and experimentation $(R R=1.78,95 \% C I$ : $1.42 ; 2,23)$ and tobacco use in the previous 30 days $(R R=2.38,95 \% C I: 1.64 ; 3.45)$. Adolescents with IBP showed greater risk of having tried tobacco $(R R=1.54,95 \% C I: 1.22 ; 1.93)$ and of having used tobacco products in the previous 30 days $(R R=$ 1.92, 95\%CI: 1.31; 2.83). Adolescents with EIBP showed greater risk of trying $(R R=2.24,95 \% C I$ : 1.57 ; 3.21$)$ and consuming tobacco products in the previous days $(R R=3.01,95 \% \mathrm{CI}: 1.63 ; 5.56)$, and lower risk of having tried alcohol at 15 years of age $(R R=0.72,95 \%$ CI: 0.55; 0.94). Public health measures aimed at reducing behavior problems in early adolescence can help reduce tobacco and alcohol consumption at 15 years.

Behavior; Mental Health; Alcoholism; Smoking; Street Drugs

\section{Resumen}

Este estudio investigó la relación entre los problemas de comportamiento externalizantes (PCE), internalizantes (PCI), y los que se producían concomitantemente (PCEI), al comienzo de la adolescencia (11 años) y el consumo de sustancias como: bebidas alcohólicas, tabaco y drogas ilícitas a los 15 años, utilizando datos de la cohorte de nacimientos de Pelotas, Brasil, de 1993. Los problemas de comportamiento fueron evaluados por el Strengths and Difficulties Questionnaire $(S D Q)$, aplicado a las madres cuando los hijos tenían 15 años. El consumo de sustancias se evaluó mediante un cuestionario anónimo, realizado por el propio adolescente. La asociación entre problemas de comportamiento y el uso de sustancias se analizó mediante la regresión de Poisson, con un ajuste robusto de variancia. Tras el ajuste para los factores de confusión, los adolescentes con PCE tuvieron un riesgo mayor de consumo abusivo de alcohol $(R R=1,74$; IC95\%: 1,$21 ; 2,50) y$ de probar estas sustancias $(R R=1,78$; IC95\%: 1,42; 2,23), además del consumo de tabaco en los últimos 30 días ( $R R=2,38$; IC95\%: 1,64; 3,45). Los adolescentes con PCI tuvieron un mayor riesgo de probar el tabaco ( $R R=1,54$; IC95\%: 1,22; 1,93) y de consumirlo durante los últimos 30 días $(R R=$ 1,92; IC95\%: 1,31; 2,83). Los adolescentes con PCEI presentaron un mayor riesgo de probar estas sustancias $(R R=2,24$; IC95\%: 1,57; 3,21) y consumir tabaco durante los últimos 30 días $(R R=$ 3,01; IC95\%: 1,63; 5,56), y menor riesgo de probar el alcohol a los 15 años ( $R R=0$, 72; IC95\%: 0,55; $0,94)$. Se requieren acciones de salud pública que actúen en la reducción de los problemas de comportamiento al principio de la adolescencia, puesto que podrían disminuir el consumo de tabaco y bebidas alcohólicas a los 15 años.

Conducta: Salud Mental; Alcoholismo; Fumar; Drogas Ilícitas
Recebido em 25/Nov/2017

Versão final reapresentada em 19/Mar/2018 Aprovado em 06/Abr/2018 